placebo has yet been published. Furthermore, patients with "confirmed persistent disease" (which was determined only after the administration of iodine-131) were removed from the final study analysis. This is notable when one considers how best to design a placebo-based investigation, since such patients may not have been identified without the radioiodine-ablation dose. Although we strongly support the goal of eliminating unnecessary radiotherapy for patients with low-risk thyroid cancer, high-quality study planning and monitoring and appropriate follow-up will be vital in the determination of how best to achieve this goal.

\section{Erik K. Alexander, M.D. \\ P. Reed Larsen, M.D.}

Brigham and Women's Hospital

Boston, MA

Since publication of their article, the authors report no further potential conflict of interest.

DOI: 10.1056/NEJMc1206712

\title{
Secondary Prevention after Ischemic Stroke
}

TO THE EDITOR: In their Clinical Practice article, Davis and Donnan (May 17 issue) ${ }^{1}$ cite two studies as supporting the use of angiotensin-converting-enzyme (ACE) inhibitors in the secondary prevention of stroke. ${ }^{2,3}$ However, in the Perindopril Protection against Recurrent Stroke Study (PROGRESS), monotherapy with perindopril lowered systolic blood pressure by $4.9 \mathrm{~mm} \mathrm{Hg}$ and diastolic blood pressure by $2.8 \mathrm{~mm} \mathrm{Hg}$, but the relative risk reduction of recurrent stroke was only $5 \%$ and not significant. ${ }^{2}$ In the Heart Outcomes Prevention Evaluation (HOPE) trial subgroup analysis, which included 1013 patients with a previous history of cerebrovascular disease, ramipril reduced systolic blood pressure by $3.1 \mathrm{~mm} \mathrm{Hg}$ and diastolic blood pressure by $1.7 \mathrm{~mm} \mathrm{Hg}$, but the relative risk reduction of recurrent stroke of $15 \%$ was not significant. ${ }^{3}$ Our previous meta-analysis ${ }^{4}$ that included 10 randomized, controlled trials showed that the pooled odds ratio for the prevention of stroke recurrence was 0.63 (95\% confidence interval [CI], 0.54 to 0.73) for trials involving diuretics as a component of therapy and 0.93 (95\% CI, 0.87 to 1.01) for trials in which treatment included ACE inhibitors. Thus, the prevention of stroke recurrence should not be considered a compelling indication for treatment with ACE inhibitors.

Haifeng Zhang, M.D., Ph.D.

First Affiliated Hospital of Nanjing Medical University Nanjing, China

Franz H. Messerli, M.D.

St. Luke's-Roosevelt Hospital Center New York, NY

Jan A. Staessen, M.D., Ph.D.

University of Leuven

Leuven, Belgium

jan.staesssen@med.kuleuven.be
Dr. Messerli reports receiving consulting fees from Novartis, Daiichi Sankyo, Pfizer, Takeda, Abbott, PharmApprove, Gilead, Servier, Bayer, and Medtronic and grant support from Forest Laboratories and Boehringer Ingelheim. No other potential conflict of interest relevant to this letter was reported.

1. Davis SM, Donnan GA. Secondary prevention after ischemic stroke or transient ischemic attack. N Engl J Med 2012;366:191422.

2. PROGRESS Collaborative Group. Randomised trial of a perindopril-based blood-pressure-lowering regimen among 6,105 individuals with previous stroke or transient ischaemic attack. Lancet 2001;358:1033-41. [Errata, Lancet 2001;358:1556, 2002;359: 2120.]

3. The Heart Outcomes Prevention Evaluation Study Investigators. Effects of an angiotensin-converting-enzyme inhibitor, ramipril, on cardiovascular events in high-risk patients. $\mathrm{N}$ Engl J Med 2000;342:145-53. [Errata, N Engl J Med 2000;342:748, 1376.] 4. Liu L, Wang $Z$, Gong $L$, et al. Blood pressure reduction for the secondary prevention of stroke: a Chinese trial and a systematic review of the literature. Hypertens Res 2009;32:1032-40.

DOI: $10.1056 / N E J M c 1207080$

TO THE EDITOR: We generally agree with the authors' discussion of this complex and controversial topic but disagree with their statement that "the longer-term health effects of stroke outweigh those of myocardial infarction." The impact of a perioperative myocardial infarction was not adequately emphasized. In the Carotid Revascularization Endarterectomy versus Stenting Trial (CREST), ${ }^{1}$ rates of the primary end point (stroke, myocardial infarction, or death from any cause during the periprocedural period or any ipsilateral stroke within 4 years after randomization) did not differ between patients at standard surgical risk who were randomly assigned to undergo stenting or endarterectomy. More patients in the stenting group had a minor stroke, whereas more patients in the endarterectomy group had a myocardial infarction. During 4 years of follow-up, among patients who had a perioperative myocar- 
dial infarction, the risk of death increased by a factor of 3.5, as compared with those who did not have a myocardial infarction, whereas patients who had a minor stroke did not have increased mortality, as compared with those who did not have a minor stroke. ${ }^{2}$ Recently published guidelines, endorsed by 14 professional societies, ${ }^{3}$ concluded that stenting is an alternative to endarterectomy in symptomatic patients with severe stenosis of the internal carotid artery, such as the patient described in the vignette of the article.

Michael R. Jaff, D.O.

Massachusetts General Hospital

Boston, MA

mjaff@partners.org

Christopher J. White, M.D.

Ochsner Clinic Foundation

New Orleans, LA

Dr. Jaff reports serving as an unpaid advisor for Abbott Vascular, Cordis, Covidien, and Medtronic and serving on the board of directors of VIVA Physicians, a nonprofit education and research organization. No other potential conflict of interest relevant to this letter was reported.

1. Brott TG, Hobson RW II, Howard G, et al. Stenting versus endarterectomy for the treatment of carotid-artery stenosis. N Engl J Med 2010;363:11-23. [Errata, N Engl J Med 2010;363:194, 498.]

2. Gray WA, Simonton CA, Verta P. Overview of the 2011 Food and Drug Administration Circulatory Devices Panel Meeting on the ACCULINK and ACCUNET Carotid Artery Stent System. Circulation 2012;125:2256-64.

3. Brott TG, Halperin JL, Abbara S, et al. 2011 ASA/ACCF/ AHA/AANN/AANS/ACR/ASNR/CNS/SAIP/SCAI/SIR/SNIS/SVM/SVS guideline on the management of patients with extracranial carotid and vertebral artery disease: executive summary: a report of the American College of Cardiology Foundation/American Heart Association Task Force on Practice Guidelines, and the American Stroke Association, American Association of Neuroscience Nurses, American Association of Neurological Surgeons, American College of Radiology, American Society of Neuroradiology, Congress of Neurological Surgeons, Society of Atherosclerosis Imaging and Prevention, Society for Cardiovascular Angiography and Interventions, Society of Interventional Radiology, Society of NeuroInterventional Surgery, Society for Vascular Medicine, and Society for Vascular Surgery developed in collaboration with the American Academy of Neurology and Society of Cardiovascular Computed Tomography. J Am Coll Cardiol 2011;57:1002-44. [Erratum, J Am Coll Cardiol 2011;57:2380.]

DOI: 10.1056/NEJMc1207080

THE AUTHORS REPLY: Zhang et al. question our interpretation of the trials of blood-pressure lowering in secondary stroke prevention. In PROGRESS, ${ }^{1}$ participants who were hypertensive or normotensive with a history of stroke or transient ischemic attack (TIA) were randomly assigned to receive perindopril or placebo; indapamide could be added to the active therapy at the discretion of the clinician. Therapy with an ACE inhibitor (with or without indapamide) resulted in a significant reduction in the subsequent risk of stroke. As noted, the combination resulted in a greater reduction in blood pressure than perindopril alone, and perindopril alone did not significantly reduce the risk of stroke. The HOPE trial ${ }^{2}$ showed a significant reduction in vascular end points in a highrisk population; this finding was consistent across a number of predefined subgroups, including those with previous stroke or TIA. Our discussion focused on the importance of blood pressure as a modifiable risk factor for stroke, and we maintain that these trials support blood-pressure reduction for secondary prevention as a general policy, at all baseline blood-pressure levels. We recommended the combination of an ACE inhibitor and a diuretic for the patient in the vignette whom we described (not ACE-inhibitor monotherapy) on the basis of PROGRESS but stated that other approaches would be reasonable.

With respect to the comments of Jaff and White: we would note that in CREST, ${ }^{3}$ major and minor stroke, but not periprocedural myocardial infarction, were associated with significant decrements in physical health (as assessed on the physical component scale of the Medical Outcomes Study 36-Item Short-Form Health Survey) at 1 year. The rates of myocardial infarction in CREST were higher than in the European trials comparing stenting with endarterectomy, reflecting the use of more sensitive criteria for diagnosis. In meta-analyses, ${ }^{4}$ carotid endarterectomy was associated with higher rates of myocardial infarction and cranial-nerve injury but lower rates of stroke and death than was stenting. Although the risk of death associated with myocardial infarction may be less than for minor stroke, major stroke confers a substantial risk of death. We pointed out that there appears to be equipoise in patients under 70 years of age.

Stephen M. Davis, M.D.

Royal Melbourne Hospital

Parkville, VIC, Australia

stephen.davis@mh.org.au

Geoffrey A. Donnan, M.D.

Florey Neuroscience Institutes

Parkville, VIC, Australia

Since publication of their article, the authors report no further potential conflict of interest.

1. PROGRESS Collaborative Group. Randomised trial of a perindopril-based blood-pressure-lowering regimen among 6,105 individuals with previous stroke or transient ischaemic attack. Lancet 2001;358:1033-41. [Errata, Lancet 2001;358:1556, 2002; 359:2120.] 
2. The Heart Outcomes Prevention Evaluation Study Investigators. Effects of an angiotensin-converting-enzyme inhibitor, ramipril, on cardiovascular events in high-risk patients. N Engl J Med 2000;342:145-53. [Errata, N Engl J Med 2000;342:748, 1376.]

3. Brott TG, Hobson RW II, Howard G, et al. Stenting versus endarterectomy for treatment of carotid-artery stenosis.
N Engl J Med 2010;363:11-23. [Errata, N Engl J Med 2010;363: 194, 498.]

4. Economopoulos KP, Sergentanis TN, Tsivgoulis G, Mariolis $\mathrm{AD}$, Stefanadis C. Carotid artery stenting versus carotid endarterectomy: a comprehensive meta-analysis of short-term and long-term outcomes. Stroke 2011;42:687-92.

DOI: 10.1056/NEJMc1207080

\section{Lecture Halls without Lectures}

TO THE EDITOR: In the Perspective article by Prober and Heath (May 3 issue), ${ }^{1}$ the authors' account of innovation in medical education through "sticky" (comprehensible and memorable) lessons emphasizes the need to enhance the quality and meaningfulness of medical education in order for the field to progress. It reminds us that there is a great divide between evidence-based educational strategies and current practice. However, the proposal is somewhat shortsighted regarding the changes that are required to bring medical education into the modern era.

The medical-education community has struggled for decades to make the training of future physicians "stickier." By no means is the problem one of awareness; instead, it is a problem of implementation. Educators face constant barriers to making teaching a priority. Education continues to be an underfunded and underappreciated aspect of academic medicine; any person completing a medical degree is somehow deemed qualified to be an educator. The literature is filled with calls to make medical education more enriching and engaging. However, until steps are taken to encourage implementation through effective teacher training and enhanced funding, reality will continue to lag behind the rhetoric.

Matthew J. Stull, M.D.
University of Cincinnati College of Medicine
Cincinnati, OH
mjstull1012@gmail.com
Robbert J. Duvivier, B.Sc.
Maastricht University
Maastricht, the Netherlands
Elizabeth Wiley, M.D., J.D.
American Medical Student Association
Sterling, VA
Drs. Stull and Wiley report being nonpaid board members of
the American Medical Student Association. No other potential
conflict of interest relevant to this letter was reported.
1. Prober CG, Heath C. Lecture halls without lectures - a pro-
posal for medical education. N Engl J Med 2012;366:1657-9.
DOI: 10.1056/NEJMc1206639

TO THE EDITOR: Prober and Heath propose "flipping" the medical school classroom by having students review didactic material (videos) before class and using face-to-face time to discuss patient cases and case-based problems to help students apply knowledge and increase the effectiveness and stickiness of the curriculum. These principles have long been recognized as best practices that shift the emphasis from teaching to learning and have been incorporated in approaches such as problem-based learning ${ }^{1}$ and team-based learning. ${ }^{2}$ For example, the Cleveland Clinic Lerner College of Medicine of Case Western Reserve University has had a "no lecture" education philosophy since its inception in 2004.

We must go beyond making learning sticky; we must help our students become self-regulated lifelong learners. ${ }^{3}$ Because accessible information grows exponentially and the half-life of knowledge continues to shrink, it is critical for students to become good lifelong learners. Doing so requires less dependence on standardized tests for assessment that measure retention of quickly outdated information and more on useful formative feedback and competency-based reflective portfolios. ${ }^{4}$ These approaches will help our students become self-regulated, self-motivated practitioners of the future.

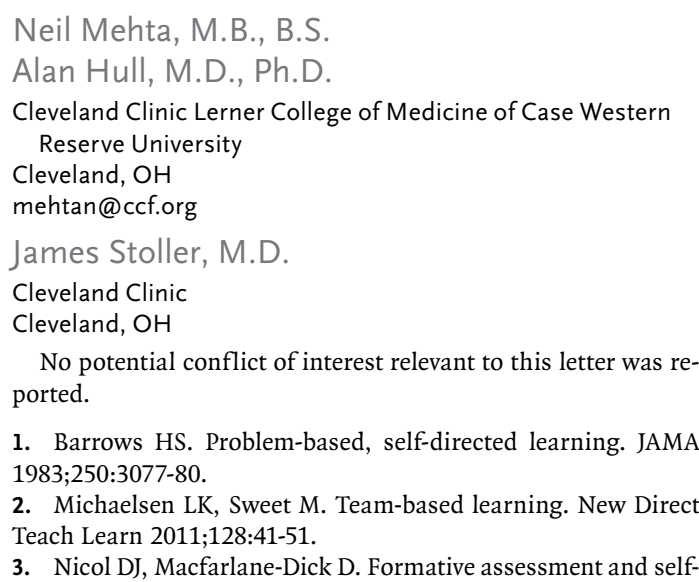

